\title{
Role of innate cytokines in mycobacterial infection
}

\author{
AM Cooper ${ }^{1}$, KD Mayer-Barber ${ }^{2}$ and A Sher ${ }^{2}$
}

\begin{abstract}
Cells of the innate immune system produce cytokines and lipid mediators that strongly influence the outcome of mycobacterial infection. In the case of Mycobacterium tuberculosis, the lung is a critical site for this interaction. Here, we review current information on the role of the major innate cytokine pathways both in controlling initial infection as well as in promoting and maintaining adaptive T-cell responses that mediate host resistance or immunopathology. Understanding this important feature of the host-pathogen interaction can provide major insights into the mechanisms of virulence and can lead to new approaches for immunological intervention in tuberculosis and other mycobacterial diseases.
\end{abstract}

\section{INTRODUCTION}

Although host defense mechanisms against mycobacteria are on the whole poorly understood, cytokines have been firmly established to have a major role in determining the outcome of infection with these important intracellular pathogens. ${ }^{1}$ The critical evidence derives from both studies in experimental models and observations on patients with genetic or drug-induced deficiencies in cytokines or their signaling pathways. Of particular note are tumor necrosis factor (TNF) and interferon (IFN)- $\gamma$ signaling the functions of which in host resistance to Mycobacterium tuberculosis (Mtb) have been well documented in both mouse models and infected humans. ${ }^{1-3}$

Cytokines have an important role in the adaptive immune response as both effectors and regulators of mycobacterial immunity, and their expression profile in CD4 $+\mathrm{T}$ cells clearly delineates the dominant Th1-like response that is associated with control of infection. This review will focus on a different, and we believe equally, important function of cytokines, their critical involvement in innate defense against mycobacterial infection, and in determining the subsequent adaptive T-cell response. The cytokines in question are produced by cells of the innate system and act both in the early stages of infection to initiate the immune response and at later stages to sustain and regulate it. Delineating their function and mechanisms of action during mycobacterial infection is thus important for understanding how host resistance is induced, maintained, and regulated. At the same time, learning how these pathogens evade or manipulate innate cytokine responses has provided critical insights into the nature of mycobacterial virulence.
Live attenuated, and heat-killed mycobacteria, are well known for their immunostimulatory properties and have been used both experimentally and clinically as adjuvants for immunization (e.g., complete Freund's adjuvant) and in immunotherapy (e.g., bladder cancer) ${ }^{4-6}$ The ability of mycobacterial products to trigger innate cytokines is considered to underlie the potency of these interventions. Delineating the role of innate cytokines in the immunostimulatory activities of mycobacteria is thus another area of major interest for this field.

The following article provides a brief overview of the roles of cytokines produced during the innate response to mycobacteria in both host defense and regulation of adaptive immunity. Owing to its global importance as a human pathogen, Mtb is the primary focus of our review, although findings from studies on other mycobacterial species will be cited where relevant. The lung is both the major site of infection for Mtb and a critical mucosal organ wherein an uncontrolled immune response can easily disrupt essential physiological function. Hence, this review will deal extensively with the induction and regulation of protective innate responses at that tissue site. Both the major innate pathways and the related mediators triggered by mycobacteria and their regulatory interactions are discussed.

\section{IL-12, IL-23, AND IL-17}

Mtb is a potent inducer of the interleukin (IL)-12p40 subunit cytokine, and deficiency in this cytokine leads to susceptibility to mycobacterial disease in humans. ${ }^{7}$ This cytokine can be expressed as a homodimer or as a heterodimer with either the IL-12p35 or the IL-23p19 subunits, which signal through the IL-12 receptor $\beta 1$ chain (IL-12R $\beta 1$ ) in conjunction with the

\footnotetext{
${ }^{1}$ Trudeau Institute, Saranac Lake, New York, USA. ${ }^{2}$ Laboratory of Parasitic Diseases, Immunobiology Section, NIAID, NIH, Bethesda, Maryland, USA. Correspondence: AM Cooper (acooper@trudeauinstitute.org) or A Sher (asher@niaid.nih.gov)

Received 12 January 2011; accepted 11 February 2011; published online 23 March 2011. doi:10.1038/mi.2011.13
} 
IL-12R 32 or the IL-23R, respectively. ${ }^{8}$ IL-12p40 is induced and rapidly expressed in response to Mtb by ligation of Tolllike receptor (TLR) 9 in vivo or TLR2 and TLR9 in vitro. ${ }^{9}$ In the mouse model, early production of IL-12p40 is not accompanied by substantial IL-23p19 or IL-12p35, but the homodimer of IL-12p40 (IL-12p80 hereafter) can drive immature dendritic cells to develop a migratory phenotype and to activate naive $\mathrm{T}$ cells in the draining lymph node ${ }^{10}$ (Figure 1). There is also an alternative splice variant of the IL-12R $\beta 1$, which is induced in dendritic cells by mycobacteria and which augments the ability of IL-12p80 to drive dendritic cell migration and T-cell activation. ${ }^{11} \mathrm{IL}-12 \mathrm{p} 80$ has also been shown to restore the lost delayed hypersensitivity response in IL-12p35/IL-12p40-deficient mice. ${ }^{12}$ Although the ability of IL-12p 80 to affect cell migration and delayed-type hypersensitivity cannot be assessed directly in the human lungs, the alternative splice variant is induced in human peripheral blood cells by mycobacteria ${ }^{11}$ and IL-12p 80 has also been identified in bronchoalveolar lavage of patients with asthma, ${ }^{13}$ suggesting that this cytokine could have a role in the initiation of cellular events in the human lung exposed to tuberculosis (TB). Indeed mutations in the IL-12p40 receptor IL-12RB1 are strongly associated with susceptibility to TB. ${ }^{14}$

IL-12p40 can be induced and expressed by itself, whereas IL-23p19 and IL-12p35 require covalent binding to IL-12p40 to be secreted, and the secretion of these cytokines in turn depends on the ligation of different pattern-recognition receptors. ${ }^{15}$ In particular, IL-23 can be made by human phagocytes exposed to Mtb quite readily, likely through the ligation of NOD2 and TLR2, but production of IL-12p70 requires that the phagocytes see another signal such as IFN- $\gamma$ or the TLR7/8 ligand R848 15 (Figure 1). A recent study using virulent $\mathrm{H} 37 \mathrm{Rv}$ and avirulent H37Ra Mtb isolates to stimulate human alveolar macrophages has shown that H37Rv markedly increases IL-23p19 but neither induces $\mathrm{p} 35$ and that IL-23 but not IL-12p70 protein is made. ${ }^{16}$ These data suggest that IL-23 is likely to be the initial IL-12p40containing heterodimer induced by $\mathrm{Mtb}$ in the human lung.

The expression of IL-12p70 in the lungs is not high, and it is likely that the local production of this cytokine within the draining lymph node is required to initiate the optimal acquired IFN- $\gamma$ response, critical for control of infection. ${ }^{17}$ Indeed the absence of IL-12p35 (and thus the loss of IL-12p70) results in a significant reduction in antigen-specific IFN- $\gamma$-producing T cells. Interestingly, cells that do make IFN- $\gamma$ in the absence of IL-12p70 are dependent on IL-23, but this IL-23-dependent response is not sufficient to maintain long-term control of bacterial growth ${ }^{17,18}$ (Figure 1). In further support for a continued protective role for IL-12p70 throughout infection is the fact that it is required to maintain pulmonary Th1 effector function. ${ }^{19}$ Importantly, although IL-12p80 alone can promote T-cell activation, it fails entirely to promote the expression of IFN- $\gamma$, and this is likely why the control of bacterial growth in the IL-12p40deficient mouse is similar to that seen in the mouse lacking both IL-12p70 and IL-23. ${ }^{11,18}$

The location of action of IL-12p40-related cytokines varies. IL-12p80 likely acts very early within the infected tissue, whereas IL-12p70 acts within the lymph node. In turn, IL-23 is likely to act both in the tissue and in the lymph node to drive the acquired response and later in the chronically inflamed tissue to promote the expression of a polarized phenotype by infiltrating lymphocytes and promoting pulmonary tissue integrity. As IL-23 is produced very early by alveolar macrophages, it is well placed to drive an early IL-17 response from innate cells within the lung, which has been shown to be important for control of acute bacterial infections. ${ }^{20}$ It is clear that after a high-dose exposure to Mycobacterium bovis BCG in the lung, there is a strong IL-17 response that comes from the $\gamma \delta$ T-cell population and that this is required for a rapid inflammatory response and for protection in this model ${ }^{21,22}$ (Figure 1). IL-17 is also expressed by $\gamma \delta \mathrm{T}$ cells in low-dose aerosol infections, ${ }^{23}$ and the IL-17 response to Mtb is largely dependent on IL-23 and IL-1 (Khader et al. ${ }^{18} \mathrm{KMB}$ and AS, unpublished data). However, in contrast to the high-dose model, IL-17 does not have a protective role in the low-dose infection model. How this relates to the kind of exposures seen in nature is unclear, but it would be interesting to compare the role of IL-17 in high- vs. low-incidence areas.

The local expression of IL-12p70 within the lungs may have a role in the expression of immunity, but it seems that a balance between this cytokine and IL-23 is likely required to maintain the mononuclear cell-enriched nature of the inflammatory response to Mtb. It has long been known that repeated exposure to high levels of mycobacterial antigen in Mtb-infected hosts can lead to development of a detrimental purulent inflammation ${ }^{24}$ known as the Koch phenomenon. It seems that both IL-23 and IL-17 are required for this response, which is characterized by a change from a mononuclear to a granulocytic inflammation in the mouse model, with the local expression of IL-23 at the inflamed site being likely responsible for the enhanced IL-17 response and change in chemokine profile that results in granulocytic influx ${ }^{25}$ (Figure 1). The differentiation state of phagocytes in the lung will also affect the cytokine profile as human monocytes differentiated by granulocyte/macrophage colonystimulating factor produce IL-23, whereas those differentiated in the presence of monocyte-stimulating factor produce IL-10 after mycobacterial exposure. ${ }^{26}$

\section{THE IL-1 CYTOKINE FAMILY}

The major IL- 1 family cytokines IL- $1 \alpha$, IL-1 $\beta$, IL- 18 , and IL-33 have potent but diverse immunological activities in inflammation and immune response regulation. Gene-knockout (KO) studies supporting a role for these cytokines in host resistance to mycobacteria first appeared a decade ago. ${ }^{27-30}$ Ironically, interest in the IL-1 family of cytokines faded around the same time that researchers began investigating the interaction between TLRs and mycobacteria. Most TLRs and receptors for the IL- 1 family members share a common adaptor molecule, MyD88. When MyD88 was shown by several groups to be critical for mouse resistance to both Mtb and Mycobacterium avium, this was interpreted as important evidence for the role of TLRs in innate recognition of these pathogens. ${ }^{31-34}$ However, more recent studies have revealed that mice deficient in the receptor for IL-1 $\alpha$ and IL-1 $\beta$ (IL-1R1) exhibit an acute susceptibility phenotype nearly indistinguishable from that of 


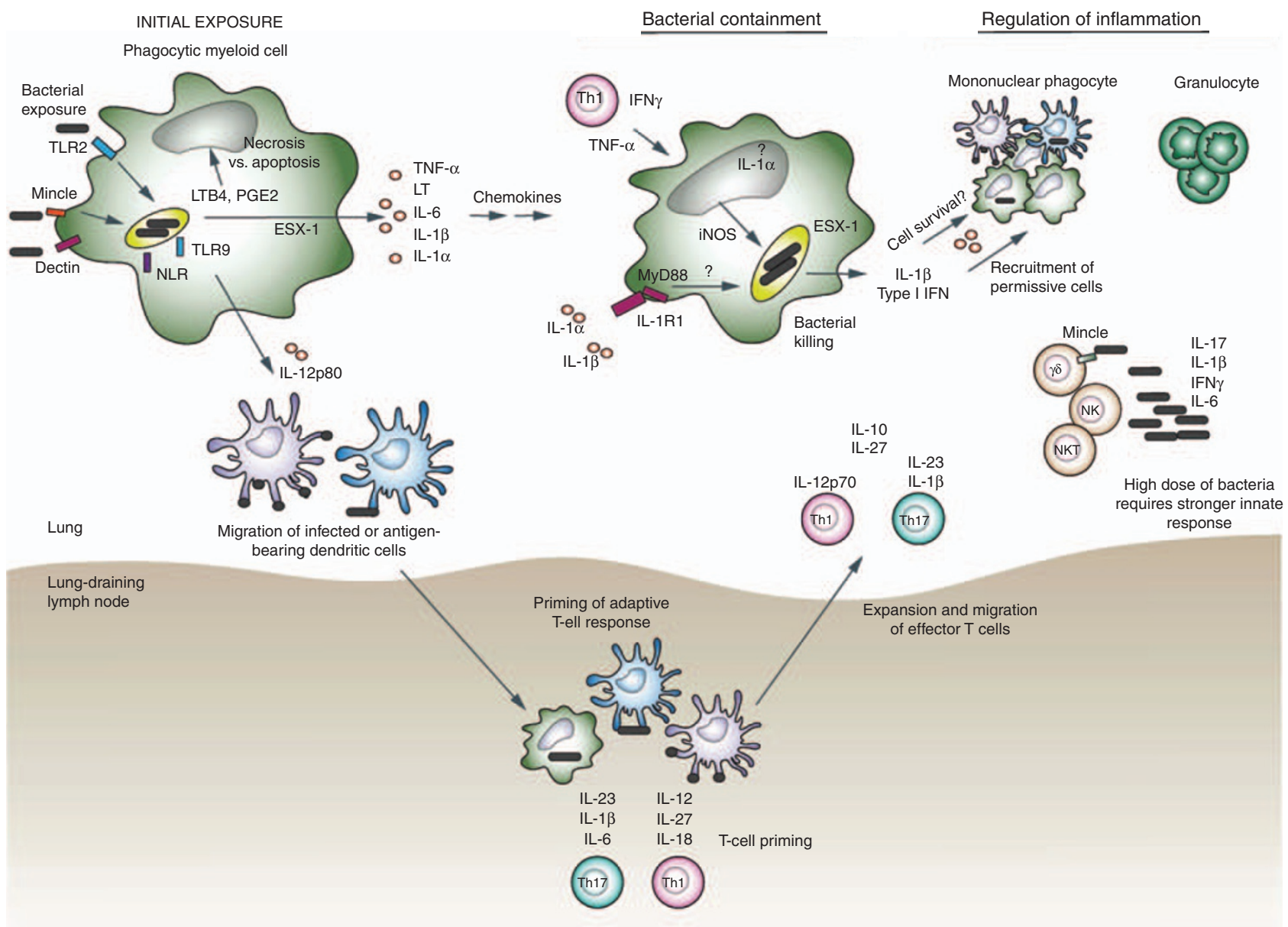

Figure 1 Innate cytokines act during the initiation, expansion, and chronic stages of Mtb infection. The exposure of lung phagocytes to invading Mtb results in binding of the bacteria and/or bacterial products to specific receptors, which then induce innate cytokine production by the infected cell. TNF and LT are produced by bacterially exposed phagocytes and promote the expression of chemokines that recruit inflammatory cells to the lung. Mtb can modulate the level of TNF and inflammation induced by altering the structure of glycolipids and mycolates on their surface. IL-12p80 promotes migration of bacteria- and antigen-bearing dendritic cells from the lung to the lymph node to start the acquired response. Virulent bacteria modulate the response of phagocytes by induction of LTB4 and promote necrotic death of infected cells. Expression of innate cytokines within the lymph node defines the effector function of T cells. Within the inflammatory site, infected phagocytes control bacterial growth in an IFN- $\gamma-$, TNF-, and IL-1R1-dependent manner. ESX-1-related activity by the bacteria can promote the protective IL-1 $\beta$ response while increasing the detrimental type I IFN response. Mononuclear inflammation is promoted by IFN- $\gamma$ and IL-12p70 while granulocytic and damaging inflammation is driven by IL-23 and IL-17 during the chronic phase of the disease. IL-10 and IL-27 regulate protective responses but also limit inflammatory responses. A high dose of bacteria or infection in the absence of acquired immunity illustrates the potential protective role of innate cytokines that do not show a role in low-dose aerosol infections. IFN- $\gamma$, interferon- $\gamma$; IL, interleukin; LT, lymphotoxin; Mtb, Mycobacterium tuberculosis; TNF, tumor necrosis factor.

MyD88-deficient mice ${ }^{35,36}$ (Figure 1).Thus, IL-1R signaling is sufficient to explain the requirement for MyD88 in the control of mycobacterial infection.

Although the field is now in general agreement about the importance of IL-1/IL-1R1 signaling in host resistance to Mtb, there is still debate about the function of IL-18 which is also MyD88 dependent and is known to specifically amplify Th1 responses. Although most reports ${ }^{29,35-38}$ document either a minor or no contribution of IL-18 and/or its receptor in the control of Mtb infection and host survival, a recent study documented acute susceptibility to Mtb equivalent to that of MyD88 KO mice in IL-18 but not in IL-18R KO mice. ${ }^{39,40} \mathrm{IL}-33$ is an IL-1 family cytokine associated with Th2 responses and therefore would be expected to have a limited role in determining host resistance to mycobacterial infection. Indeed infected mice deficient in $s t 2$, the signaling chain of the IL-33R, were found to be indistinguishable from wild-type (WT) control animals in their bacterial loads and pulmonary histopathology. ${ }^{41}$

The role of IL-1 in Mtb infection was first documented using mice doubly deficient in IL- $1 \alpha$ and IL-1 $\beta$ or in IL-1R1 signaling. ${ }^{28,30}$ All studies involving IL-1R1 mice reported decreased survival and increased pulmonary bacterial loads. These findings were confirmed in the two more recent reports, ${ }^{35,36}$ which directly compared IL-1R1 KO and MyD88 KO mice and described indistinguishable necrotic lung pathology and susceptibility to infection (Figure 1). It is noteworthy that none of the aforementioned studies observed major defects in the TNF $\alpha$, IL-12/IFN $\gamma$, or inducible nitric oxide synthase pathways 
in the absence of IL-1R1 signaling, arguing that IL-1 regulates host resistance to Mtb by a distinct mechanism not involving the former effectors previously demonstrated as essential for control of infection.

Mice singly deficient in IL-1 $\beta$ have also been found to be acutely susceptible to Mtb infections, arguing not only that IL- $1 \beta$ is required for host resistance but also that IL- $1 \beta$ has a non-redundant function that cannot be compensated for by IL- $\alpha$, which signals through the same IL-1R1 complex. ${ }^{36}$ IL-1 $\beta$ is a potent pyrogenic cytokine the biological activity of which must be tightly regulated. This is achieved through various mechanisms including control of gene expression, post-transcriptional activation of the cytokine through protein cleavage, and through inhibition of receptor binding by interaction with decoy receptors and soluble IL-1R antagonists. ${ }^{42}$ The proteolytic cleavage step from the inactive $31 \mathrm{kDa}$ pro-form to the active-form $17 \mathrm{kDa}$ IL- $1 \beta$ is mediated by a multiprotein complex known as the inflammasome, which triggers the activation of caspase-1, the enzyme that converts immature to mature IL- $1 \beta .{ }^{42}$

Bone marrow-derived macrophages and dendritic cells exposed to Mtb in vitro produce mature IL- $1 \beta$ through the nod like receptor family, pyrin domain containing 3 (NLRP3)inflammsome-mediated activation of caspase- $1 .{ }^{37,43,44}$ This production has been shown to be dependent on the presence of the mycobacterial ESAT-6 secretion system 1 (ESX-1) secretion system, which mediates release of major virulence factors encoded by the region of difference $1(\mathrm{RD}-1)$ region of the $\mathrm{Mtb}$ genome $^{44}$ (Figure 1). The apoptosis-associated speck-like protein containing a caspase recruitment domain (ASC) is a critical component of the NLRP 3 inflammasome and as a consequence, ASC-deficient bone marrow-derived macrophages and dendritic cells fail to produce IL- $1 \beta$ in response to Mtb exposure in vitro. ${ }^{36,37,43,44}$ Importantly, although all studies agree that the NLRP3 inflammasome through ASC and caspase- 1 is critical for IL-1 $\beta$ induction in vitro, they also show that mice deficient in caspase-1, ASC, and NALP3 display little or no phenotype after low-dose aerosol infection with Mtb. ${ }^{36,37,43}$ The fact that IL-1 $\beta$ deficient mice are highly susceptible, whereas casapse-1-, ASC-, and NLRP3-deficient mice are not, argues that IL-1 $\beta$ can be cleaved in vivo by an inflammasome-independent mechanism. Candidate cleavage enzymes include other caspases, chymases, cathepsins, and elastases. ${ }^{42}$ The latter two are expressed in high levels by neutrophils, which have been previously shown to produce and process IL- $1 \beta$ by a caspase- 1 -independent pathway. ${ }^{45}$ Identification of the cellular source of the IL- $1 \beta$ produced in vivo in the lungs of infected mice is required to study the respective roles of this and other candidate IL- $1 \beta$ cleavage pathways and more importantly to determine the general effector function of this cytokine in the cellular response to Mtb. It is of further interest that in addition to IL- $1 \beta$ single-deficient mice, animals deficient only in IL- $1 \alpha$ are also highly susceptible to Mtb infection (KMB and AS, in preparation), and that IL- $1 \alpha$ in contrast to IL-1 $\beta$ does not require proteolytic activation and can also function as a nuclear transcription factor. Thus, another fascinating issue concerns how these two innate cytokine ligands for IL-1R1 cooperate in the induction of host resistance against Mtb.
Perhaps the key question raised by the dramatic susceptibility of IL-1-deficient mice to Mtb infection concerns the mechanism by which IL-1 restricts bacterial growth in non-deficient hosts. Although the available literature argues that IL-1 has little or no effect on the adaptive Th1 response, we have observed a major defect in Th17 responses in deficient mice (KMB and AS, unpublished). Nevertheless, despite the absence of IL-17, no defects in pulmonary neutrophil recruitment have been observed in IL-1R1 KO animals (Sugawara et al. ${ }^{28} ; \mathrm{KMB}$ and AS, unpublished). As noted above, high levels of necrosis are observed in the lungs of infected IL-1R-deficient mice, and hence, one possibility is that IL-1 regulates host resistance by modulating cell death (Figure 1).

In humans, a role for IL-1 signaling in host resistance to Mtb is supported by a number of genetic studies demonstrating an association of polymorphisms in the $I L-1$ or $I L-1 R$ genes with altered disease progression and susceptibility. ${ }^{46-49}$ IL-1 blockade has become an accepted therapy for many auto-inflammatory diseases and rheumatoid arthritis, and if indeed IL-1 signaling is important for control of Mtb in humans, there is a potential risk for disease exacerbation or reactivation of latent infection in Mtb-exposed individuals undergoing such treatment. However, a survey of the literature revealed only one case report of Mtb reactivation associated with IL-1 inhibition through Anakrina. ${ }^{50}$ With the advent of newer, more effective approaches for IL-1 blockade and the increased use of these therapeutic agents, studies should be initiated to determine whether IL-1 has a critical role in the control of human Mtb infection analogous to that previously revealed by cytokine blockade for TNF. ${ }^{51}$

\section{TYPE I ANDTYPE II IFNS}

IFNs have long been recognized as important for host resistance to mycobacterial infection and considered as potential therapeutics. However, it is only in the last decade that IFN production by the innate immune system has been considered as an important component of this response. In the case of type II IFN $-\gamma$, which has a well-established protective function against Mtb and other mycobacteria in both mice and humans, conventional CD4 + and CD8 + T cells were considered the primary source of the cytokine. It is now known that the innate lymphocytes, $\gamma \delta \mathrm{T}$ cells, natural killer (NK) T cells, and NK cells that possess restricted or invariant receptor repertoires can also produce IFN- $\gamma$ in response to mycobacterial stimulation and can display protective effects against Mtb both in vitro and in vivo upon adoptive transfer ${ }^{1}$ (Figure 1). However, whether these cells contribute to the IFN- $\gamma$-dependent control of infection when an adaptive $\mathrm{T}$-cell response is also present remains unclear. For example, although depletion experiments indicate that NK cell-produced IFN- $\gamma$ is responsible for the partial resistance of $\mathrm{rag}^{-I-}$ mice to Mtb infection, ${ }^{52}$ similar depletion of NK cells in T cell-sufficient WT mice fails to influence control of infection. ${ }^{53}$ Nevertheless, NK cells, $\gamma \delta$ T cells, and NK T cells may serve as secondary sources of IFN- $\gamma$ that prevent the adaptive immune system from being overwhelmed as a consequence of heavy or hypervirulent mycobacterial exposure (Figure 1). 
In addition, it is possible that these innate sources of the cytokine have a more important protective role in humans than in mice, particularly in the setting of T-cell immunodeficiency as seen in $\mathrm{HIV}^{+}$individuals infected with Mtb.

In contrast to IFN- $\gamma$, there is a lack of consensus as to whether type I IFN has any anti-mycobacterial activity, and indeed most studies suggest that these cytokines actually promote infection. In mouse $M$. avium infection, the continuous infusion of IFN- $\beta$ led to increased resistance as evidenced by a 10 -fold reduction in hepatic and splenic bacterial loads. ${ }^{54}$ However, in Mtb infection, endogenous type I IFN promotes rather than limits bacterial growth. ${ }^{55-57}$ In particular, the hypervirulence of an Mtb strain has been correlated with enhanced type I IFN synthesis, which was associated with impaired Th1 immune responses. ${ }^{55}$ Later, it was shown that type I IFN receptor-deficient mice, chronically infected with various different Mtb strains, displayed significantly reduced bacterial loads when compared with similarly infected WT animals. ${ }^{58}$ More recently, it was reported that Mtb-infected mice treated with the type I IFN inducer polyinosinic-polycytidylic acid stabilized with poly-L-lysine show exacerbated lung pathology and mycobacterial burden and, in the case of chronically infected animals administered the drug, reactivated disease. ${ }^{58}$ This type I IFN-induced exacerbation was not associated with altered T-cell function, but instead correlated with the recruitment into the lungs of a population of $\mathrm{CD} 11 \mathrm{~b}^{+} \mathrm{F} 4 / 80^{+} \mathrm{Gr} 1^{\text {int }}$ myeloid cells that display decreased major histocompatibility complex II and IFN- $\gamma$ R expression, as well as enhanced bacterial levels relative to the same subset of cells purified from infected, untreated control mice ${ }^{58}$ (Figure 1). Thus, in mouse TB, as previously described for Listeria infection in mice, ${ }^{59}$ type I IFN enhances infection.

The association of type I IFN induction with Mtb virulence has also been observed at the level of the infected cell. Thus, comparison of gene expression profiles in mouse macrophages infected with virulent Mtb vs. an avirulent mutant with an inactive ESX-1 secretion system, revealed the selective induction of type I IFN-associated genes and IFN- $\beta$ by the virulent, but not the avirulent, bacteria ${ }^{60}$ (Figure 1). This virulence-associated type I IFN response was found to be independent of the TLR adaptor TRIF and RIP2, the adaptor for NOD1 and NOD2 signaling, but required TBK1, a kinase also necessary for type 1 IFN induction by viruses and Listeria. ${ }^{60}$ Although partially confirming the above findings, a second study differed in arguing that Nod1, Nod2, and RIP2 are indeed required for type I IFN induction by virulent Mtb and proposed that the function of ESX-1 in virulence determination is to provide bacterial Nod ligands (e.g., $\mathrm{N}$-acetyl-muramyl dipeptide) access to these host cytoplasmic receptors through damage of the phagosomal vacuolar membrane. ${ }^{61}$

A recent report has suggested that type I IFN induction may also be a factor determining the outcome of human Mtb infection. ${ }^{62}$ The authors surveyed gene expression in whole blood collected from a large group of patients with latent and active TB. They found that most patients with active disease displayed an expression signature dominated by IFN-induced genes in neutrophils (Figure 1). The same profile was also present in $\sim 10 \%$ of asymptomatic patients with latent infection, suggesting that these individuals represent the subgroup at the highest risk for active TB. Taken together, the above findings implicate type I IFN as a downregulator of protective immune function in both mouse and human Mtb infections. Both the pathways for induction of type I IFN by Mtb and the mechanism(s) by which these cytokines suppress host resistance are currently active areas of investigation in the field.

\section{IL-10, IL-27, AND IL-6}

Both IL-27 and IL-6 are related, ${ }^{63}$ and these cytokines along with IL-10 modulate the response to mycobacteria. IL-10 is elevated in samples obtained from patients with $\mathrm{TB},{ }^{64}$ and an increased ability to innately produce IL-10 is associated with increased incidence of disease. ${ }^{65} \mathrm{IL}-10$ reduces the protective response to Mtb in animal models but only when it is strongly induced i.e., in the case of the CBA mouse wherein IL- 10 is produced by phagocytes within the lung lesion and reduces expression of TNF and IL-12p $40 .{ }^{66,67} \mathrm{IL}-10$ has also recently been shown to block phagosome maturation in human alveolar macrophages. ${ }^{68}$ The importance of IL-10 may be double edged; however, as even in mouse strains in which it does not limit the protective response, it seems to be required for long-term control of inflammation ${ }^{69}$ (Figure 1).

IL- 6 is a potent cytokine that is induced by Mtb but which has varying effects dependent on the nature of the challenge. In the low-dose aerosol model, its absence leads to delayed expression of the IFN- $\gamma$ response in the lung and a modest increase in bacterial burden. ${ }^{70}$ Indeed the T-cell response seems to need IL-6 to develop optimally during mycobacterial infections and vaccination $^{71,72}$ (Figure 1). Similar to the case for IL-17, low-dose aerosol infection does not require IL-6 to be controlled, whereas in the absence of IL- 6 , a high dose of bacteria is lethal, ${ }^{73}$ suggesting that the ability of IL- 6 to augment the response becomes critical when bacterial burden is high (Figure 1). This similar dose dependency of susceptibility between IL- 6 and IL-17R KO mice suggests that it will be important to determine whether the key action of IL-6 in the high-dose model is to promote Th17. In addition to acting during initiation of responses, IL- 6 expression in the inflammatory site can modulate the maintenance of IL-17-producing cells. ${ }^{74}$ The relative levels of IL- 6 and transforming growth factor- $\beta$ could also determine the Treg/Th17 ratio within the inflammatory site, thereby influencing the inflammatory and protective outcomes during chronic disease.

Both IL-6 and IL-27 signal through the gp130 receptor. Absence of this receptor on LysM-expressing cells results in increased expression of inflammatory cytokines and phagocyte activation, as well as in increased Th17 responses but does not result in increased control of bacterial growth or pathology. ${ }^{75}$ The absence of the IL-27 receptor (IL-27R) leads to better control of bacterial growth in the lung, but the mechanism behind this is unclear. ${ }^{76,77} \mathrm{IL}-27$ promotes IL-10 and antagonizes IFN$\gamma$ and IL-17 responses, ${ }^{78,79}$ and alteration in these cytokines will substantially alter the development of the inflammatory response and expression of protective immunity. Recent studies have also identified a role for the IL-27p28 subunit alone 
as an antagonist of gp130 signaling that reduces IL-6-induced IL-17 and IL-10 production. ${ }^{80}$ Determining how IL-27R activity reduces protection against $\mathrm{Mtb}$ is an important task not only as a pathway to improve vaccination strategies but also because it reflects the response of the host to a chronic pathogen and may indicate a pathway whereby the host tolerates the pathogen to protect the organ infected. In this regard, it is critical to note that although bacterial burden is reduced in the absence of this receptor, mice have greater inflammatory responses and reduced survival $^{76,77}$ (Figure 1).

\section{LT/TNF}

The relative roles of TNF and lymphotoxin (LT) in Mtb have been a subject of controversy recently. Although it is clear that the absence of TNF results in rapid susceptibility to Mtb growth and death of the host ${ }^{81}$ the specific reason for this enhanced susceptibility is not completely clear. Phagocyte activation is a key deficiency in the absence of TNF, but there is also a serious deficiency in chemokine expression and the influx of cells to the granuloma in the absence of this cytokine ${ }^{82}$ (Figure 1). TNF that is restricted to the membrane can contain an acute infection, but the full complement of TNF activity is required for long-term maintenance of the mononuclear granuloma and control of bacterial growth. ${ }^{83,84}$ In the absence of LT, there is a loss of lymph nodes, and hence, bone marrow chimeras lacking both secreted LT- $\alpha$ and the membrane-bound LT- $\beta$ have been used to assess the role of these molecules. In particular, mice lacking both membrane-bound and secreted LT can express an acquired response, but the organization of the granuloma is compromised and these mice are more susceptible to disease. ${ }^{85}$ In contrast, mice lacking just the membrane-associated LT- $\beta$ are no more susceptible than are WT mice to an aerosol infection, ${ }^{85}$ but have a modestly increased susceptibility to disease when infected through the intranasal route. ${ }^{86}$ In contrast to earlier data, a recent paper has suggested that LT- $\alpha$ does not have a significant role in acute susceptibility to Mtb, rather an increase in bacterial burden late in infection is reported. ${ }^{87}$ Although the authors of the latter paper suggest that earlier LT- $\alpha$ mice had defective TNF production, this was not the case for the earlier study wherein equivalent TNF production between WT and LT- $\alpha$-deficient mice was reported ${ }^{85}$ Furthermore, it has been shown that the absence of either TNF or LT- $\alpha$ has distinct consequences on the progression of Mycobacterium leprae infections in the mouse model..$^{88}$ It may be that the relative levels of LT and TNF are critical for host defense, and that dose, route, and possibly bacterial isolate may have a role; this is an area that still needs to be clarified.

\section{EICOSANOIDS}

Eicosanoids are lipid mediators that are derived from arachidonic acid and include prostaglandins, lipoxins, and leukotrienes. In the context of TB, the balance between prostaglandin E2 and lipoxin A4 production by Mtb-infected macrophages has been shown to affect the outcome of infection both in vitro and in vivo by directing macrophages either toward apoptosis or necrosis, respectively. ${ }^{89}$ The apoptotic cell death program is considered to be induced by attenuated Mtb, which leads to control of mycobacterial growth inside the infected macrophage and is accompanied by prostaglandin E2 synthesis, whereas virulent Mtb promotes necrosis, which is associated with high leukotriene B4 and lipoxin A4 and favors bacterial replication ${ }^{89}$ (Figure 1). Accordingly, mice deficient in 5-lipogygenase, which metabolizes arachidonic acid into lipoxin A4 and leukotriene $\mathrm{B} 4$, are more resistant to aerosol infection with $\mathrm{Mtb}$ and harbor fewer bacteria in the lung, ${ }^{90}$ whereas mice lacking prostaglandin E synthase display higher bacterial loads and succumb to infection earlier. ${ }^{91,92}$ Moreover, a recent study has revealed a conserved role for leukotriene A4 hydrolase in both zebrafish and humans in regulating the balance between pro and antiinflammatory eicosanoids during mycobacterial infection..$^{93}$ Taken together, these findings reveal a striking function for eicosanoids that spans various host species. Proinflammatory cytokines such as TNF are likely to be key cues directly linking the innate immune system to the eicosanoid pathway during Mtb infection.

\section{BACTERIAL INDUCTION OF INNATE CYTOKINES}

The ability of the innate cytokines to affect not only the initiation but also the expression of acquired immunity and the potential for a pathological response makes them a target for manipulation by the bacteria. The importance of this manipulation is twofold: first, the bacteria need to limit immunity and second they need to promote the inflammation that allows for liquefaction of the granuloma and generation of the infectious cough. The ability of the bacteria to modulate expression of innate cytokines has recently been assessed not only for lab strains but also for the increasingly appreciated variety of clinical isolates. It is now clear that Mtb migrated out of Africa with the human population and has separated into several groups since that time. ${ }^{94}$ In particular, a subset of the successful Beijing strains express a phenolic glycolipid that is associated with decreased induction of cytokines such as IL-10, IL-6, and TNF relative to other clinical isolates and the laboratory strain $\mathrm{H} 37 \mathrm{Rv}$, which do not express this glycolipid ${ }^{95,96}$ (Figure 1). In addition, a recent study characterizing both the human and genetic diversity of patients suggests that the innate autophagic response to TB is effective but only in those patients infected with strains that are unable to make the phenolic glycolipid, and thereby fail to limit the innate response..$^{97}$ In addition to the phenolic glycolipid, modest changes in other major Mtb molecules are critical for virulence as illustrated by the fact that a mutant Mtb that is unable to cyclopropanate $\alpha$-mycolates is killed in vivo in a TNF-dependent manner. ${ }^{98}$ Conversely, the inability to trans-cyclopropanate methoxy- and keto-mycolates increases the inflammatory activity of the mutant and the trehalose dimycolate extracted from it, suggesting that the bacteria fine tunes this major surface molecule to modulate innate immunity and inflammation ${ }^{99}$ (Figure 1). Trehalose dimycolate is now known to bind the $\mathrm{FcR} \gamma$-associated, C-type lectin Mincle and signal through SykCARD9-Bcl10-Malt 1 to promote the release of IL-1, IL-6, and TNF, and accordingly the cytokine response to whole bacteria is reduced in the absence of Mincle. ${ }^{100-102}$ Defining how the 
bacteria initiate and modulate the innate cytokine response will provide essential information for understanding the interaction between host and pathogen, as well as the potential for defined mycobacterial products to be used as adjuvants.

\section{CONCLUSIONS}

The importance of the innate response to Mtb has been overlooked in the past because of the apparent overriding role of the acquired response in limiting this disease. Unlike the acquired response, it seems that the role of the innate cytokine response in survival is more dependent on route, dose, and isolate, and these variables need to be considered when interpreting the importance of a specific cytokine. In addition, there may be fundamental differences between the innate response between different animal models and humans not least because of the phagocyte populations that are most commonly examined. In this review, we have outlined the major innate cytokine pathways that influence the outcome of mycobacterial infection but have not discussed the equally important topic of how these pathways might regulate each other and/or cooperate in mediating host resistance. This is an exciting and challenging new research area that is likely to be a major focus for future work in the field. In addition, it is of great interest to determine the extent to which both the lung and gut commensal flora affect the expression of innate cytokine responses with regard to this chronic pulmonary infection.

Our improved comprehension of the role of the innate response in inducing, maintaining, and regulating the acquired response suggests that modulating the innate arm of the immune system could aid in control of Mtb disease. In addition, understanding the function of innate cytokines in this persistent bacterial infection will provide more general insights into how the host responds to a chronic stimulus and to the pathogenesis of chronic inflammatory diseases of other causes.

\section{ACKNOWLEDGMENTS}

We thank Dan Barber, Carl Feng, Bruno Andrade, Alena Srinivasan, David Kugler, Bernadette Saunders, and Alexei Tumanov for helpful discussion. AS and KMB are supported by the intramural research program of the NIAID. AMC is supported by the Trudeau Institute $\mathrm{NIH}$ grants Al67723, Al69121, and Al46530 and an American Lung Association De Souza award.

\section{DISCLOSURE}

The authors declared no conflict of interest.

(C) 2011 Society for Mucosal Immunology

\section{REFERENCES}

1. Cooper, A.M. \& Khader, S.A. The role of cytokines in the initiation, expansion, and control of cellular immunity to tuberculosis. Immunol. Rev. 226, 191-204 (2008).

2. Harris, J. \& Keane, J. How tumour necrosis factor blockers interfere with tuberculosis immunity. Clin. Exp. Immunol. 161, 1-9 (2010).

3. Zhang, S. et al. Inborn errors of interferon (IFN)-mediated immunity in humans: insights into the respective roles of IFN-alpha/beta, IFN-gamma, and IFN-lambda in host defense. Immunol. Rev. 226, 29-40 (2008).

4. Grange, J.M., Bottasso, O., Stanford, C.A. \& Stanford, J.L. The use of mycobacterial adjuvant-based agents for immunotherapy of cancer. Vaccine 26, 4984-4990 (2008).
5. Freund, J. et al. Antibody formation and sensitization with the aid of adjuvants. J. Immunol. 60, 383-398 (1948).

6. Alexandroff, A.B., Nicholson, S., Patel, P.M. \& Jackson, A.M. Recent advances in bacillus Calmette-Guerin immunotherapy in bladder cancer. Immunotherapy 2, 551-560 (2010).

7. Filipe-Santos, O. et al. Inborn errors of IL-12/23- and IFN-gammamediated immunity: molecular, cellular, and clinical features. Semin. Immunol. 18, 347-361 (2006).

8. Trinchieri, G., Pflanz, S. \& Kastelein, R. The IL-12 family of heterodimeric cytokines: new players in the regulation of T cell responses. Immunity 19 , 641-644 (2003).

9. Bafica, A., Scanga, C., Feng, C., Leifer, C., Cheever, A. \& Sher, A. TLR9 regulates Th1 responses and cooperates with TLR2 in mediating optimal resistance to Mycobacterium tuberculosis. J. Exp. Med. 202, 1715-1724 (2005).

10. Khader, S. et al. Interleukin $12 \mathrm{p} 40$ is required for dendritic cell migration and T cell priming after Mycobacterium tuberculosis infection. J. Exp. Med. 203, 1805-1815 (2006).

11. Robinson, R. et al. Mycobacterium tuberculosis infection induces il12rb1 splicing to generate a novel IL-12R $\beta 1$ isoform that enhances DC migration. J. Exp. Med. 207, 591-605 (2010).

12. Holscher, C. et al. A protective and agonistic function of IL-12p40 in mycobacterial infection. J. Immunol. 167, 6957-6966 (2001).

13. Walter, M., Kajiawara, N., Karanja, P., Castro, M. \& Holtzman, M.J. Interleukin-12 p40 production by barrier epithelial cells during airway inflammation. J. Exp. Med. 193, 339-351 (2001).

14. Altare, F. et al. Impairment of mycobacterial immunity in human interleukin-12 receptor deficiency. Science 280, 1432-1435 (1998).

15. Gerosa, F. et al. Differential regulation of interleukin 12 and interleukin 23 production in human dendritic cells. J. Exp. Med. 205, 1447-1461 (2008).

16. Silver, R. et al. Human alveolar macrophage gene responses to Mycobacterium tuberculosis strains H37Ra and H37Rv. Am. J. Respir. Cell Mol. Biol. 40, 491-504 (2009).

17. Cooper, A.M., Kipnis, A., Turner, J., Magram, J., Ferrante, J. \& Orme, I.M. Mice lacking bioactive IL-12 can generate protective, antigenspecific cellular responses to mycobacterial infection only if the IL-12 p40 subunit is present. J. Immunol. 168, 1322-1327 (2002).

18. Khader, S. et al. IL-23 compensates for the absence of IL-12p70 and is essential for the IL-17 response during tuberculosis but is dispensable for protection and antigen-specific IFN-gamma responses if IL-12p70 is available. J. Immunol. 175, 788-795 (2005).

19. Feng, C. et al. Maintenance of pulmonary Th1 effector function in chronic tuberculosis requires persistent IL-12 production. J. Immunol. 174, 4185-4192 (2005).

20. Riol-Blanco, L. et al. IL-23 receptor regulates unconventional IL-17producing T cells that control bacterial infections. J. Immunol. 184, 1710-1720 (2010).

21. Umemura, M. et al. IL-17-mediated regulation of innate and acquired immune response against pulmonary Mycobacterium bovis bacille Calmette-Guerin infection. J. Immunol. 178, 3786-3796 (2007).

22. Okamoto Yoshida, Y. et al. Essential role of IL-17A in the formation of a mycobacterial infection-induced granuloma in the lung. J. Immunol. 184, 4414-4422 (2010).

23. Lockhart, E., Green, A. \& Flynn, J. IL-17 production is dominated by gammadelta T cells rather than CD4 T cells during Mycobacterium tuberculosis infection. J. Immunol. 177, 4662-4669 (2006).

24. Koch, R. Fortsetzung der mittheilung uber ein heilmittel gegen tuberkulose. Dtsch. Med. Wochenschr. 17, 101 (1891).

25. Cruz, A. et al. Pathological role of Interleukin 17 in mice subjected to repeated BCG vaccination after infection with Mycobacterium tuberculosis. J. Exp. Med. 207, 1609-1616 (2010).

26. Verreck, F.A.W. et al. Human IL-23-producing type 1 macrophages promote but IL-10-producing type 2 macrophages subvert immunity to (myco)bacteria. Proc. Natl Acad. Sci. USA 101, 4560-4565 (2004).

27. Juffermans, N.P. et al. Interleukin-1 signaling is essential for host defense during murine pulmonary tuberculosis. J. Infect. Dis. 182, 902-908 (2000).

28. Sugawara, I., Yamada, H., Hua, S. \& Mizuno, S. Role of interleukin (IL)-1 type 1 receptor in mycobacterial infection. Microbiol. Immunol. 45, 743750 (2001).

29. Sugawara, I., Yamada, H., Kaneko, H., Mizuno, S., Takeda, K. \& Akira, S. Role of interleukin-18 (IL-18) in mycobacterial infection in IL-18-genedisrupted mice. Infect. Immunol. 67, 2585-2589 (1999). 
30. Yamada, H., Mizumo, S., Horai, R., Iwakura, Y. \& Sugawara, I. Protective role of interleukin-1 in mycobacterial infection in IL-1 alpha/beta doubleknockout mice. Lab. Invest. 80, 759-767 (2000).

31. Sugawara, I., Yamada, H., Mizuno, S., Takeda, K. \& Akira, S. Mycobacterial infection in MyD88-deficient mice. Microbiol. Immunol. 47, 841-847 (2003).

32. Scanga, C., Bafica, A., Feng, C., Cheever, A., Hieny, S. \& Sher, A. MyD88-deficient mice display a profound loss in resistance to Mycobacterium tuberculosis associated with partially impaired Th1 cytokine and nitric oxide synthase 2 expression. Infect. Immunol. 72, 2400-2404 (2004).

33. Feng, C. et al. Mice lacking myeloid differentiation factor 88 display profound defects in host resistance and immune responses to Mycobacterium avium infection not exhibited by Toll-like receptor 2 (TLR2)- and TLR4-deficient animals. J. Immunol. 171, 4758-4764 (2003).

34. Fremond, C., Yeremeev, V., Nicolle, D., Jacobs, M., Quesniaux, V. \& Ryffel, B. Fatal Mycobacterium tuberculosis infection despite adaptive immune response in the absence of MyD88. J. Clin. Invest. 114, 1790-1799 (2004).

35. Fremond, C.M. et al. IL-1 receptor-mediated signal is an essential component of MyD88-dependent innate response to Mycobacterium tuberculosis infection. J. Immunol. 179, 1178-1189 (2007).

36. Mayer-Barber, K.D. et al. Cutting edge: caspase-1 independent IL-1 \{beta\} production is critical for host resistance to Mycobacterium tuberculosis and does not require TLR signaling in vivo. J Immunol. 184, 3326-3330 (2010).

37. Walter, K., Holscher, C., Tschopp, J. \& Ehlers, S. NALP3 is not necessary for early protection against experimental tuberculosis. Immunobiology 215, 804-811 (2010).

38. Kinjo, Y. et al. Contribution of IL-18 to Th1 response and host defense against infection by Mycobacterium tuberculosis. J. Immunol. 169, 323-329 (2002).

39. Schneider, B.E. et al. A role for IL-18 in protective immunity against Mycobacterium tuberculosis. Eur. J. Immunol. 40, 396-405 (2010).

40. Korbel, D.S., Schneider, B.E. \& Schaible, U.E. Innate immunity in tuberculosis: myths and truth. Microbes Infect. 10, 995-1004 (2008).

41. Wieland, C.W., van der Windt, G.J., Florquin, S., McKenzie, A.N. \& van der Poll, T. ST2 deficient mice display a normal host defense against pulmonary infection with Mycobacterium tuberculosis. Microbes Infect. 11, 524-530 (2009).

42. Dinarello, C.A. Immunological and inflammatory functions of the interleukin-1 family. Annu. Rev. Immunol. 27, 519-550 (2009).

43. McElvania Tekippe, E. et al. Granuloma formation and host defense in chronic Mycobacterium tuberculosis infection requires PYCARD/ASC but not NLRP3 or caspase-1. PLoS One 5, e12320 (2010).

44. Koo, I.C., Wang, C., Raghavan, S., Morisaki, J.H., Cox, J.S. \& Brown, E.J. ESX-1-dependent cytolysis in lysosome secretion and inflammasome activation during mycobacterial infection. Cell Microbiol. 10, 1866-1878 (2008).

45. Greten, F.R. et al. NF-kappaB is a negative regulator of IL-1beta secretion as revealed by genetic and pharmacological inhibition of IKKbeta. Cell 130, 918-931 (2007).

46. Bellamy, R., Ruwende, C., Corrah, T., McAdam, K.P.W.J., Whittle, H.C. \& Hill, A.V. Assessment of the interleukin-1 gene cluster and other candidate gene polymorphisms in host susceptibility to tuberculosis. Tuberc. Lung Dis. 79, 83-89 (1998).

47. Gomez, L.M., Camargo, J.F., Castiblanco, J., Ruiz-Narvaez, E.A., Cadena, J. \& Anaya, J.M. Analysis of IL1B, TAP1, TAP2 and IKBL polymorphisms on susceptibility to tuberculosis. Tissue Antigens 67, 290-296 (2006).

48. Awomoyi, A.A. et al. Polymorphism in IL1B: IL1B-511 association with tuberculosis and decreased lipopolysaccharide-induced IL-1 beta in IFN-gamma primed ex-vivo whole blood assay. J. Endotoxin. Res. 11, 281-286 (2005).

49. Wilkinson, R.J. et al. Influence of polymorphism in the genes for the interleukin (IL)-1 receptor antagonist and IL-1beta on tuberculosis. J. Exp. Med. 189, 1863-1874 (1999).

50. Settas, L.D., Tsimirikas, G., Vosvotekas, G., Triantafyllidou, E. \& Nicolaides, P. Reactivation of pulmonary tuberculosis in a patient with rheumatoid arthritis during treatment with $\mathrm{LL}-1$ receptor antagonists (anakinra). J. Clin. Rheumatol. 13, 219-220 (2007).
51. Taylor, P. \& Feldmann, M. Anti-TNF biologic agents: still the therapy of choice for rheumatoid arthritis. Nat. Rev. Rheumatol. 5, 578-582 (2009).

52. Feng, C.G. et al. NK cell-derived IFN-gamma differentially regulates innate resistance and neutrophil response in $\mathrm{T}$ cell-deficient hosts infected with Mycobacterium tuberculosis. J. Immunol. 177, 7086-7093 (2006).

53. Junqueira-Kipnis, A.P. et al. NK cells respond to pulmonary infection with Mycobacterium tuberculosis, but play a minimal role in protection. J. Immunol. 171, 6039-6045 (2003).

54. Denis, M. Recombinant murine beta interferon enhances resistance of mice to systemic Mycobacterium avium infection. Infect. Immunol. 59, 1857-1859 (1991).

55. Manca, C. et al. Virulence of a Mycobacterium tuberculosis clinical isolate in mice is determined by failure to induce Th1 type immunity and is associated with induction of IFN-alpha/beta. Proc. Natl Acad. Sci. USA 98, 5752-5757 (2001).

56. Manca, C. et al. Hypervirulent M. tuberculosis W/Beijing strains upregulate type I IFNs and increase expression of negative regulators of the Jak-Stat pathway. J. Interferon Cytokine Res. 25, 694-701 (2005).

57. Ordway, D. et al. The hypervirulent Mycobacterium tuberculosis strain HN878 induces a potent TH1 response followed by rapid downregulation. J. Immunol. 179, 522-531 (2007).

58. Antonelli, L.R. et al. Intranasal Poly-IC treatment exacerbates tuberculosis in mice through the pulmonary recruitment of a pathogenpermissive monocyte/macrophage population. J. Clin. Invest. 120, 1674-1682 (2010).

59. O'Connell, R.M. et al. Type I interferon production enhances susceptibility to Listeria monocytogenes infection. J. Exp. Med. 200, 437-445 (2004).

60. Stanley, S.A., Johndrow, J.E., Manzanillo, P. \& Cox, J.S. The type I IFN response to infection with Mycobacterium tuberculosis requires ESX-1mediated secretion and contributes to pathogenesis. J. Immunol. 178, 3143-3152 (2007).

61. Pandey, A.K. et al. NOD2, RIP2 and IRF5 play a critical role in the type I interferon response to Mycobacterium tuberculosis. PLoS Pathog. 5, e1000500 (2009).

62. Berry, M.P. et al. An interferon-inducible neutrophil-driven blood transcriptional signature in human tuberculosis. Nature 466, 973-977 (2010).

63. Kastelein, R., Hunter, C. \& Cua, D. Discovery and biology of IL-23 and IL-27: related but functionally distinct regulators of inflammation. Annu. Rev. Immunol. 25, 221-242 (2007).

64. Barnes, P.F., Lu, S., Abrams, J.S., Wang, E., Yamamura, M. \& Modlin, R.L. Cytokine production at the site of disease in human tuberculosis. Infect. Immunol. 61, 3482-3489 (1993).

65. Awomoyi, A., Marchant, A., Howson, J., McAdam, K., Blackwell, J. \& Newport, M. Interleukin-10, polymorphism in SLC11A1 (formerly NRAMP1), and susceptibility to tuberculosis. J. Inf. Dis. 186, 1808-1814 (2002).

66. Turner, J. et al. In vivo IL-10 production reactivates chronic pulmonary tuberculosis in C57BL/6 mice. J. Immunol. 169, 6343-6351 (2002).

67. Beamer, G. et al. Interleukin-10 promotes Mycobacterium tuberculosis disease progression in CBA/J mice. J. Immunol. 181, 5545-5550 (2008).

68. O'Leary, S., O'Sullivan, M. \& Keane, J. IL-10 blocks phagosome maturation in Mycobacterium tuberculosis-infected human macrophages. Am. J. Respir. Cell Mol. Biol. e-pub ahead of print 11 February 2011 doi:10.1165/rcmb.2010-03190c.

69. Higgins, D., Sanchez-Campillo, J., Rosas-Taraco, A., Lee, E., Orme, I. \& Gonzalez-Juarrero, M. Lack of IL-10 alters inflammatory and immune responses during pulmonary Mycobacterium tuberculosis infection. Tuberculosis (Edinb) 89, 149-157 (2009).

70. Saunders, B., Frank, A., Orme, I. \& Cooper, A. Interleukin-6 induces early gamma interferon production in the infected lung but is not required for generation of specific immunity to Mycobacterium tuberculosis infection. Infect. Immunol. 68, 3322-3326 (2000).

71. Appelberg, R., Castro, A.G., Pedrosa, J. \& Minoprio, P. Role of interleukin-6 in the induction of protective T cells during mycobacterial infections in mice. Immunology 82, 361-364 (1994).

72. Leal, I.S., Smedegard, B., Andersen, P. \& Appelberg, R. Interleukin-6 and interleukin-12 participate in induction of a type 1 protective T-cell response during vaccination with a tuberculosis subunit vaccine. Infect. Immunol. 67, 5747-5754 (1999). 
73. Ladel, C.H., Blum, C., Dreher, A., Reifenberg, K., Kopf, M. \& Kaufmann, S.H.E. Lethal tuberculosis in interleukin-6-deficient mice. Infect. Immunol. 65, 4843-4849 (1997).

74. Jones, G. et al. Loss of CD4+ T cell IL-6R expression during inflammation underlines a role for IL-6 trans signaling in the local maintenance of Th17 cells. J. Immunol. 184, 2130-2139 (2010).

75. Sodenkamp, J., Behrends, J., Förster, I., Müller, W., Ehlers, S. \& Hölscher, C. gp130 on macrophages/granulocytes modulates inflammation during experimental tuberculosis. Eur. J. Cell Biol. e-pub ahead of print 6 December 2010 dx. doi.org/10.1016/j.ejcb.2010.10.010.

76. Pearl, J.E. et al. IL-27 signaling compromises control of bacterial growth in mycobacteria-infected mice. J. Immunol. 173, 7490-7496 (2004).

77. Holscher, C. et al The IL-27 receptor chain WSX-1 differentially regulates antibacterial immunity and survival during experimental tuberculosis. J. Immunol. 174, 3534-3544 (2005).

78. Stumhofer, J. et al. Interleukin 27 negatively regulates the development of interleukin 17-producing T helper cells during chronic inflammation of the central nervous system. Nat. Immunol. 7, 937-945 (2006).

79. Stumhofer, J. et al. Interleukins 27 and 6 induce STAT3-mediated T cell production of interleukin 10. Nat. Immunol. 8, 1363-1371 (2007).

80. Stumhofer, J. et al. A role for IL-27p28 as an antagonist of gp130mediated signaling. Nat. Immunol. 11, 1119-1126 (2010).

81. Flynn, J.L. et al. Tumor necrosis factor-alpha is required in the protective immune response against Mycobacterium tuberculosis in mice. Immunity 2, 561-572 (1995).

82. Roach, D., Bean, A., Demangel, C., France, M., Briscoe, H. \& Britton, W. TNF regulates chemokine induction essential for cell recruitment, granuloma formation, and clearance of mycobacterial infection. J. Immunol. 168, 4620-4627 (2002).

83. Saunders, B., Tran, S., Ruuls, S., Sedgwick, J., Briscoe, H. \& Britton, W. Transmembrane TNF is sufficient to initiate cell migration and granuloma formation and provide acute, but not long-term, control of Mycobacterium tuberculosis infection. J. Immunol. 174, 4852-4859 (2005).

84. Olleros, M. et al. Contribution of transmembrane tumor necrosis factor to host defense against Mycobacterium bovis bacillus Calmette-guerin and Mycobacterium tuberculosis infections. Am. J. Path. 166, 1109-1120 (2005).

85. Roach, D., Briscoe, H., Saunders, B., France, M., Riminton, S. \& Britton, W. Secreted lymphotoxin- $\alpha$ is essential for the control of an intracellular bacterial infection. J. Exp. Med. 193, 239-246 (2001).

86. Ehlers, S. et al. The lymphotoxin beta receptor is critically involved in controlling infections with the intracellular pathogens Mycobacterium tuberculosis and Listeria monocytogenes. J. Immunol. 170, 5210-5218 (2003).
87. Allie, N. et al. Limited role for lymphotoxin $\alpha$ in the host immune response to Mycobacterium tuberculosis. J. Immunol. 185, 4292-4301 (2010).

88. Hagge, D. et al. Lymphotoxin-alpha and TNF have essential but independent roles in the evolution of the granulomatous response in experimental leprosy. Am. J. Path. 174, 1379-1389 (2009).

89. Divangahi, M., Desjardins, D., Nunes-Alves, C., Remold, H.G. \& Behar, S.M. Eicosanoid pathways regulate adaptive immunity to Mycobacterium tuberculosis. Nat. Immunol. 11, 751-758 (2010).

90. Bafica, A. et al. Host control of Mycobacterium tuberculosis is regulated by 5 -lipoxygenase-dependent lipoxin production. J. Clin. Invest. 115, 1601-1606 (2005).

91. Chen, M. et al. Lipid mediators in innate immunity against tuberculosis: opposing roles of PGE2 and LXA4 in the induction of macrophage death. J. Exp. Med. 205, 2791-2801 (2008).

92. Behar, S.M., Divangahi, M. \& Remold, H.G. Evasion of innate immunity by Mycobacterium tuberculosis: is death an exit strategy? Nat. Rev. Microbiol. 8, 668-674 (2010).

93. Tobin, D.M. et al. The Ita4h locus modulates susceptibility to mycobacterial infection in zebrafish and humans. Cell 140, 717-730 (2010).

94. Gagneux, S. et al. Variable host-pathogen compatibility in Mycobacterium tuberculosis. Proc. Natl Acad. Sci. USA 103, 2869-2873 (2006).

95. Reed, M. et al. A glycolipid of hypervirulent tuberculosis strains that inhibits the innate immune response. Nature 431, 84-87 (2004).

96. Wang, C. et al. Innate immune response to Mycobacterium tuberculosis Beijing and other genotypes. PLoS One 25, e13594 (2010).

97. Intemann, C. et al. Autophagy gene variant IRGM -261T contributes to protection from tuberculosis caused by Mycobacterium tuberculosis but not by M. africanum strains. PLoS Pathog. 5, e1000577 (2009).

98. Glickman, M., Cox, J. \& Jacobs, W. A novel mycolic acid synthetase is required for cording, persistence and virulence of Mycobacterium tuberculosis. Mol. Cell 5, 717-727 (2000).

99. Rao, V., Gao, F., Chen, B., Jacobs, W.J. \& Glickman, M. Transcyclopropanation of mycolic acids on trehalose dimycolate suppresses Mycobacterium tuberculosis -induced inflammation and virulence. J. Clin. Invest. 116, 1660-1667 (2006).

100. Schoenen, H. et al. Cutting edge: Mincle is essential for recognition and adjuvanticity of the mycobacterial cord factor and its synthetic analog trehalose-dibehenate. J. Immunol. 184, 2756-2760 (2010).

101. Werninghaus, K. et al. Adjuvanticity of a synthetic cord factor analogue for subunit Mycobacterium tuberculosis vaccination requires FcRgamma-Syk-Card9-dependent innate immune activation. J. Exp. Med. 206, 89-97 (2009).

102. Ishikawa, E. et al. Direct recognition of the mycobacterial glycolipid, trehalose dimycolate, by C-type lectin Mincle. J. Exp. Med. 206, 2879-2888 (2009). 